\title{
Lip sarcoma cord presenting as vaginal hydrocele
}

\author{
${ }^{1}$ Dr Abid Ali.K.P, ${ }^{2}$ Dr Asiq Sideeq,${ }^{3}$ Dr Prasad paul, ${ }^{4}$ Dr Deepu Babu \\ ${ }^{1}$ Associate professor.Dept of general surgery MES medical college \\ ${ }^{2}$ Professor of pathology MES medical college \\ ${ }^{3}$ Assistant professor Dept of General surgery MES medical college \\ ${ }^{4}$ Junior resident Dept of general surgery MES medical college
}

\begin{abstract}
Liposarcomas are neoplasm of mesodermal origin derived from adipose tissues and corresponds to 10-14\% of all soft tissue sarcomas.Paratesticular liposarcoma is very rare and only 5-7\%incidence.Because of the difficulty in determining the exact site of origin ,paratesticular liposarcoma include all liposarcoma arising in the structures around the testes and lower end of the cord.Most commonly its arising from spermatic cord.The tissue of origin is seems to be the subserous retroperitoneal fat which continuous distally with the cord .The treatment of this is still unsettled because of its rarity. Its considered as low grade malignancy compared to other paratesticular tumour.Hence high inguinal orchidectomy is cosidered as the treatment. The role of retroperitoneal dissection of lymphnode is still uncertain.Among soft tissue sarcomas liposarcoma is the most radiosensitive tumour and also there are reported cases of effective post operative radiotherapy treatment .Less than 200 cases are reported in the literature.We are presenting a case of liposarcoma presented clinically as vaginal hydrocele.
\end{abstract}

Key-words: liposarcoma; paratesticular ; high,orchidectomy; hydrocele; gemcitabine.

\section{Key Messages}

Paratesticular liposarcomas are very rare neoplasm and usually arise from the subserous fat .Compared to other paratesticular tumour its of low grade tumour and needs only high inguinal orchidectomy.Pleomorphic type is considered as the most common variety.

\section{Introduction}

Liposarcoma is a rare pathological entity. By far is the most common histological subtype of genitourinary sarcomas in adults. Liposarcoma is tumour of adults with peak incidence between ages 50 and 65 years. 20\% of soft tissue sarcomas in adults. Most common sites are thigh and retroperitoneum Approximately two hundred cases were reported in the literature. We are here by presenting a case with a typical clinical scenario of paratesticular liposarcoma.

\section{Case History:}

A 60 year old male presented with swelling of left hemiscrotum of 6 months duration with gradual increase in the size of the swelling.Clinical examination revealed a 8 × $6 \mathrm{~cm}$ fluctuant transilluminant swelling without any cough impulse on left hemiscrotum and we made hydrocele as the clinical diagnosis..Pre operative uss showed a lobulated predominantly hypoechoic lesion in relation to cord with minimal vascularity and hydrocele( fig 1)

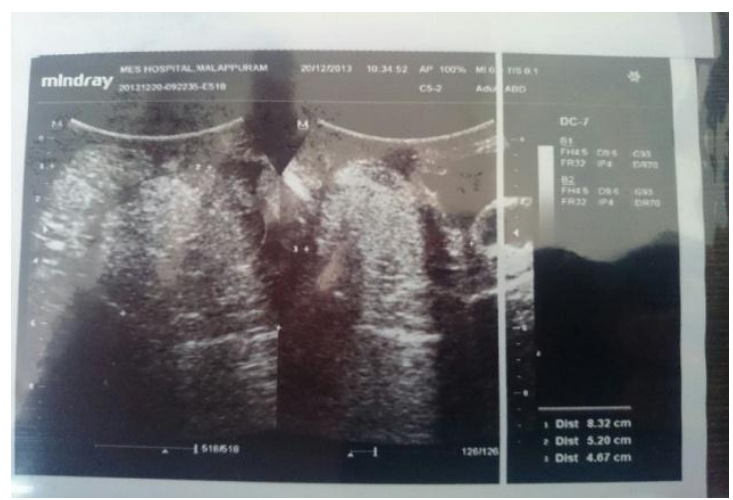


In view of this report we investigated for testicular malignancy and sent for tumour markers and found to be negative.Pre operative CT , uss chest and chest $\mathrm{x}$ ray done for mtastatic work up and found to be negative hence proceeded with high inguinal orchidectomy

\section{Histopathology}

Grossly there is a tumour of size $9.5 \times 5 \times 4.3 \mathrm{~cm}$ in the paratesticular area pale yellow lobulated fibrofattty tumour.section shows cellular neoplasm partly circumscribed in areas and with infiltrating margins in other areas composed of loosely dispersed spindle to pleomorphic cells with hyperchromatic nuclei in a background of fibroblastic stroma.Large cells with bizarre hyperchromatic nuclei and occasional cells in mitosis.Other section shows fibrocollagenous tissues and lobules of mature adipo cytes.Periphery of cellular tumour shows lobules of mature adipocytes with variation in size,occasional hyperchromatic nuclei and stromal bizarre cells.Bizarre pleomorphic cells positive for vimentin ,desmin.low proliferative index positive for KI67.Adjuscent atypical adipocyte positive for S100.Smooth cell actin negative.and gave a diagnosis of de differentiated liposarcoma sclerosing variety(FIG2,3).

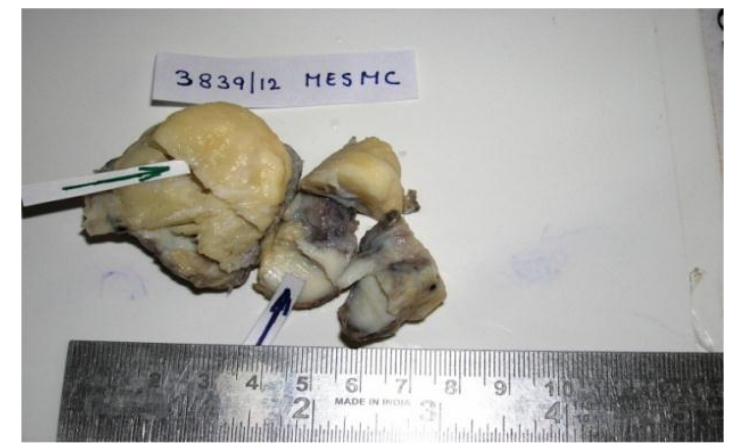

FIG 2 Gross specimen of the lesion

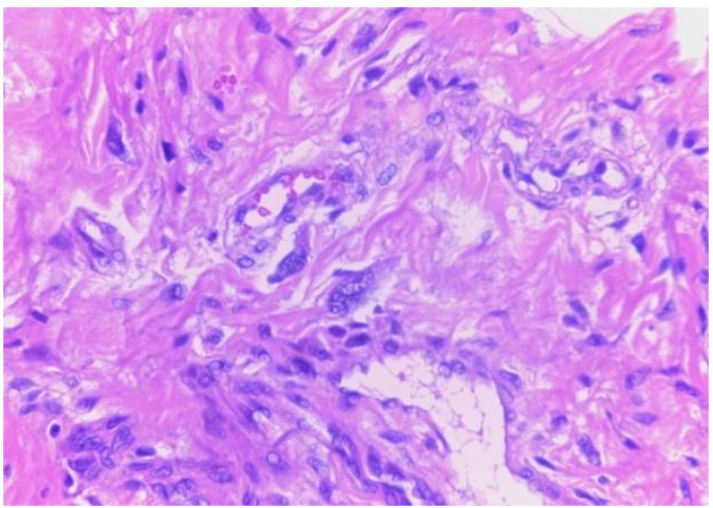

FIG 3 Microscopy of the lesion

\section{Discussion}

Liposarcoma is tumour of adults with peak incidence between ages 50 and 65 years. The youngest reported patient was 27 years old, and the oldest was 80 years ${ }^{1,2}$.Less than 200 cases were reported up to date.The common sites of occurance with order of frequency are spermatic cord ,tunica and epididymis. The presentation can be mistaken for hernia or hydrocele as in our case ${ }^{3}$. Para-testicular liposarcoma appears as a nonhomogeneous mass with variable echoes consistent with fat. CT scan with IV contrast is an excellent radiological tool for diagnosis.It is used preoperatively and postoperatively for staging and followup, respectively.

Liposarcoma can be a well differentiated ,myxoid round cell and pleomorphic one.Of which myxoid type is known to be the most common type. In differentiated liposarcoma well and poority differentiated liposarcoma and non lipomatous sarcoma coexist in one tumour. It has a vague prognosis compared to other types of sarcoma making the histological diagnosis difficult ${ }^{4.5 .6}$. On a chromosomal level, differentiated liposarcoma frequently displays the same chromosomal abnormally associated with well differentiated liposarcomas - the presence of a supernumerary ring or giant chromosome derived from the $12 \mathrm{2q}(13-21)$ region.

Hematogenous and lymphatic spread is usually a late event exhibited by high-grade tumors. With regards to treatment radical orchiectomy and en bloc removal of the tumor with wide local excision are the 
standard treatment. It has been reported that no therapeutic advantage could be attributed to superficial inguinal or retroperitoneal lymphadenectomy.

Liposarcoma in general are radiosensitive tumour but in the case of paratesicular tumour its role is less clear. Recurrence after radiotherapy may have a higher-grade and more aggressive neoplasm with dedifferentiation. Radiation therapy is recommended in addition to surgery ${ }^{5}$ in situations with evidence of tumor with propensity for more aggressive behavior (i.e., if inadequate margin, recurrence, highgrade or if there is lymphatic invasion). There is a paucity of data regarding the results of chemotherapy in metastatic paratesticular liposarcoma but the results of chemotherapy for liposarcoma at other sites are poor. Adjuvant chemotherapy has been reported in 2 cases but followup has been short. Doxorubicin chemotherapy has been used occasionally. Para-testicular liposarcoma has a high propensity for local recurrence, the commonest reason for which is incomplete resection. Pleomorphic liposarcoma appears to be somewhat sensitive to gemcitabine based and ifosfamide based chemotherapy. The reported recurrence rate ranges between $46 \%$ and $57 \%$. Prognosis and survival vary in relation to histopathological classification. Myxoid and welldifferentiated have better prognosis than round cell and pleomorphic liposarcoma. Five-year survival rates are $80 \%$ and $20 \%$, respectively

\section{References}

[1]. Bellinger MF, Gibbons MD, Koontz WW, Graff M.Paratesticular liposarcoma. J Urol. 1978;1 1:285-288.

[2]. Dreyfuss ML, Lybash S. Malignant mixed tumor of thespermatic cord. J Urol. 1940;44:314-324.

[3]. M. A. Fagundes, A. L. Zietman, A. F. Althausen et al., "Themanagement of spermatic cord sarcoma," Cancer, vol. 77, no. 9,pp. $1873-1876,1996$.

[4]. J. E. Logan, M. B. Williams, M. E. Shaves, and K. A. McCammon,"Liposarcoma of the spermatic cord: a case report andreview of management," UroToday International Journal, vol. 3,no. 4, 2010.

[5]. F. Hinman, T. E. Gibson, F. Hinman, and T. E. Gibson, "Tumorsof the epididymis, spermatic cord and testicular tunics: a reviewof literature and report of three new cases,"Arch surgery,vol.8,no.1,pp.100-137,1924.

[6]. U. Demirci, S. Buyukberber, A. Cakir Demirci, S. Buyukberber,A. Cakir et al., "Synchronous testicular liposarcoma andprostate adenocarcinoma: a case report," Cases Journal, vol. 3,p. 27, 2010

[7]. Devita, Hellman, Rosenberg, Canhcer, principles and practice of Oncology, 9e1540 - 1542

[8]. Singer S, Riedel E, Histologic subtype and margin of resection predict pattern of recurrence and survival for liposarcoma. Ann Surgery 2003, 203:358 